\title{
Influence of buffer-layer surface morphology on the self-organized growth of InAs on InP(001) nanostructures
}

\author{
L. González, a) J. M. García, R. García, F. Briones, J. Martínez-Pastor, ${ }^{\text {b) }}$ \\ and C. Ballesteros ${ }^{\mathrm{c})}$ \\ Instituto de Microelectrónica de Madrid (CNM, CSIC), Isaac Newton 8, 28760, Tres Cantos, Madrid, Spain
}

(Received 10 August 1999; accepted for publication 29 December 1999)

\begin{abstract}
We have studied the influence of InP buffer-layer morphology in the formation of InAs nanostructures grown on $\mathrm{InP}(001)$ substrates by solid-source molecular-beam epitaxy. Our results demonstrate that when InP buffer layers are grown by atomic-layer molecular-beam epitaxy, InAs quantum dot-like structures are formed, whereas InP buffer layers grown by MBE produce quantum-wire-like structures. The optical properties of these corrugated structures make them potential candidates for their use in light-emitting devices at $1.55 \mu \mathrm{m}$. (C) 2000 American Institute of Physics. [S0003-6951(00)00309-0]
\end{abstract}

InAs nanostructures grown on $\operatorname{InP}(001)$ are promising candidates for light emitting devices in the wavelength range $1.3-1.55 \mu \mathrm{m} .{ }^{1-3} \mathrm{~A}$ widely investigated technological approach is to use self-organized nanostructures that appear spontaneously as an efficient way to relax elastic strain in lattice-mismatched heteroepitaxy. In order to obtain a welldefined emission wavelength, nanostructures should have a good uniformity in size and shape. Ordering in the growth plane and a precise control of the nanostructure morphology is a key issue for self-organized systems. Due to a 3.2\% lattice mismatch of InAs on $\operatorname{InP}(001)$, elastic strain relaxation takes place above a certain critical thickness via a change of morphology from two-dimensional (2D) to threedimensional (3D). Much of the research developed on selforganized growth of InAs on InP substrates is devoted to quantum-dot (QD) formation. ${ }^{1-4}$ However, very recent results ${ }^{5}$ have shown that the chemical composition of the buffer layer (InP, InGaAs, or InAlAs) is determinant in configuring the final shape of the InAs self-organized nanostructures.

In this letter, we demonstrate that the growth conditions of the InP buffer layer also controls the surface rearrangement of the strained InAs layer grown on top. Therefore, it is possible to obtain either QD or quantum-wire (QWr) structures for identical InAs coverage and growth conditions.

There are two sets of samples grown for this work. In the first one, InAs was deposited on a 200-nm-thick InP buffer layer grown either by molecular-beam epitaxy (MBE) or by atomic-layer molecular-beam epitaxy (ALMBE) (Ref. 6) using solid sources. MBE buffer layers were grown in the $2 \times 4$ surface reconstruction at $T_{s}=460^{\circ} \mathrm{C}$ and at a beamequivalent pressure (BEP) $\left(\mathrm{P}_{2}\right)=5 \times 10^{-6}$ Torr. For the ALMBE InP buffer layers, a substrate temperature $T_{s}$ $=400{ }^{\circ} \mathrm{C}$ is used. The $\mathrm{P}_{2}$ pulsed flux reaching the surface sample is controlled by means of the reflectivity difference

\footnotetext{
${ }^{\text {a) Electronic mail: luisa@imm.cnm.csic.es }}$

b) Also at: Institut de Ciencia dels Materiales de l'Universitat de València, P.O. Box 2085, 46071-Valencia, Spain.

c) Also at: Escuela Politécnica Superior, Universidad Carlos III, c/ Butarque 15, 28911-Leganés, Madrid, Spain.
}

(RD) technique in order to optimize surface stoichiometry for growing InP planar surfaces. ${ }^{6}$ The InAs layers, 2.5 (ML) thick, were deposited at a BEP $\left(\mathrm{As}_{4}\right)=1.5-2 \times 10^{-6}$ Torr, a growth rate of $0.5 \mathrm{ML} / \mathrm{s}$, and $T_{s}=400^{\circ} \mathrm{C}$, which is chosen to minimize the P/As exchange. After InAs growth, an annealing at $480^{\circ} \mathrm{C}$ under arsenic pressure during $10-20 \mathrm{~s}$ was performed. We observe, in agreement with other authors, ${ }^{3,4}$ that InAs growth takes place in a $2 \mathrm{D}$ mode. The $2 \mathrm{D}-3 \mathrm{D}$ transition occurs during the annealing process at $T_{s}$ $=480^{\circ} \mathrm{C}$, as shown by a change into a spotty reflection high-energy electron diffraction (RHEED) pattern. The second set of samples consist of 5-, 8-, and 12-ML-thick $\mathrm{In}_{x} \mathrm{Ga}_{1-x}$ As layers with In contents $x=0.95,0.9$, and 0.85, respectively, deposited on InP buffer layers grown by MBE. We have increased the layer thickness for decreasing $x$ according to the expected dependence of critical thickness with misfit. ${ }^{?}$

As-grown samples were either immediately cooled down and taken out of the growth chamber for morphology studies by atomic-force microscopy (AFM), or covered with a 50nm-thick InP cap layer for optical and structural characterization by high-resolution transmission electron microscopy (HRTEM). Photoluminescence (PL) experiments were performed as a function of temperature from 18 to $300 \mathrm{~K}$. The signal was analyzed with a linear polarizer set in front of a $0.22 \mathrm{~m}$ SPEX single monochromator and synchronously detected by a $77 \mathrm{~K}$ cooled Ge photodetector.

Figure 1(a) shows the AFM image of an InAs/InP sample in which the InP buffer layer has been grown by ALMBE. The self-organized structures have a QD shape, whose height ranges between 1.6 and $6.8 \mathrm{~nm}$, a full width at half maximum (FWHM) $W \approx 26 \mathrm{~nm}$, and a typical density $\rho \approx 2 \times 10^{10} \mathrm{~cm}^{-2}$. On the other hand, the same amount of InAs deposited on a InP buffer layer grown by MBE gives rise to periodic undulations [Fig. 1(b)]. These QWr-like structures are oriented along the $[1 \overline{1} 0]$ direction. The height of the wires, $h$ ranges between 0.6 and $2 \mathrm{~nm}$ and the length exceeds $1 \mu \mathrm{m}$. Their FWHM $W$ is around $18 \mathrm{~nm}$ and the pitch period $\lambda$ is about $24 \mathrm{~nm}$. Meanwhile, AFM profiles over a selected QWr along the [1 $\overline{10}$ ] direction show height fluctuations below $2 \mathrm{ML}, h$ can vary up to $7 \mathrm{ML}$ between 


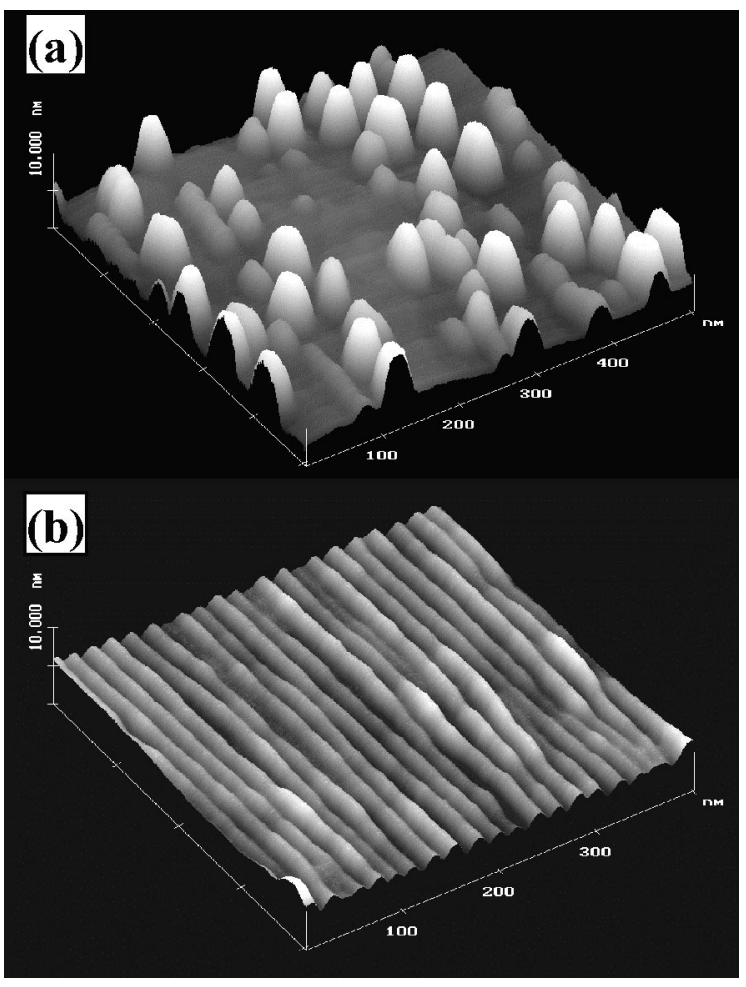

FIG. 1. AFM images of a 2.5-ML-thick InAs layer deposited on a $200 \mathrm{~nm}$ buffer layer grown by ALMBE (a) and MBE (b) on an $\operatorname{InP}(001)$ substrate.

different wires. The above given average dimensions for QD and QWr structures are consistent with the amount of deposited InAs. So, within the error introduced by the AFM estimate of the shape and even possible inaccuracies in the growth parameters, we can say that there is no significant amount of material from the buffer layer involved in the formation of the nanostructures. HRTEM images (not shown) from $\{110\}$ cross sections of capped samples show that InAs QWr-like structures remain after capping with InP. However, no clear chemical contrast between InAs and InP could be obtained under any imaging conditions.

The PL spectra of the different QWr-like samples (Fig. 2) evidence a strong light emission centered around $1.55 \mu \mathrm{m}$ at room temperature (RT), whose overall integrated intensity

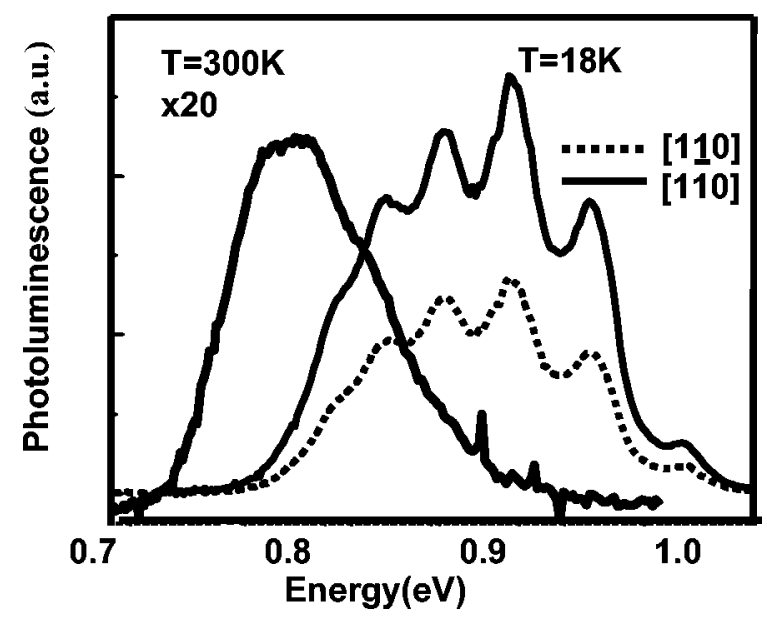

FIG. 2. PL spectra at $18 \mathrm{~K}$ and RT for InAs QWr-like structures. Emitted light is more intense when polarizer is set parallel to the wire direction $[1 \overline{1} 0]$. [1 $1 \overline{1}$ anisotropy during growth.
Downloaded 26 Feb 2010 to 161.111.180.191. Redistribution subject to AIP license or copyright; see http://apl.aip.org/apl/copyright.jsp is about 20 times less intense than that observed at low temperatures. At lower temperatures, several lines can be resolved from the PL spectra (Fig. 2). The measured linewidths of such individual lines are around $30 \mathrm{meV}$. The intensity of each individual line exhibits a different temperature evolution. When increasing temperature, the excitons jump from the smaller wires (probably by thermal scape) to the higher ones, whose PL intensity begins to decrease above $200 \mathrm{~K}^{8}{ }^{8}$ The PL peak energies agree approximately with those found in strained InAs/InP quantum wells ${ }^{2,9}$ thicker than about 3-4 $\mathrm{ML}$, and the energy separation is consistent with 1-MLthickness fluctuation. The different PL lines of the QWr-like structures can be correlated with height variations from 3 to $9 \mathrm{ML}$, in good agreement with the $h$ range found by AFM. No evidences of the existence of a wetting layer are found in our PL data, in accordance with the AFM estimate of the QWr volume and period.

Another evidence for strong lateral confinement in our QWr-like structures lies in the polarization degree of the emitted light. We observe that PL intensity is stronger when the polarizer is set parallel to the wire direction [1 10$]$, as shown in Fig. 2. The polarization degree, defined as $P$ $=\left(I_{[1 \overline{10}]}-I_{[110]}\right) /\left(I_{[1 \overline{1} 0]}+I_{[110]}\right)$, is independent of incident light polarization, and practically constant with temperature $P \approx 30 \%$.

QWr-like structures are similar in size and shape to those obtained for the InAs/InGaAs/InP system reported in Ref. 5. In our case, QD or QWr structures appear using the same InAs growth process, so we can exclude the formation of an InGaAs intermediate layer as the cause of QWr formation. The difference in the surface morphology of the InAs layer, QD, or QWr, can be only ascribed to the different growth processes of the InP buffer layer.

In strained heteroepitaxial systems, QD structures are commonly self-assembled. In the case of the QWr, we should ask about the role of the buffer layer on the QWr formation process. Nucleation of 3D features is usually assumed to be mainly controlled by elastic strain, surface energy, and surface diffusion kinetics. On one hand, strain and surface energy are characteristic magnitudes for a certain heteroepitaxial system [InAs on $\operatorname{InP}(001)$, in this case]. On the other hand, surface diffusion kinetics can be dominated by extrinsic parameters. In particular, anisotropic bufferlayer morphology can make the surface diffusion different along the [110] and [1 $\overline{10}]$ directions. In this case, the development of asymmetric morphologies during the 2D-3D transition seems a reasonable consequence.

In fact, we have observed that InP buffer layers grown by MBE systematically show an anisotropic roughness with features preferentially aligned in the [1 $\overline{10}]$ direction. Similar features have been observed in homoepitaxial growth of InP (Ref. 10) and GaAs (Ref. 11) on (001)-oriented substrates. Surface reconstruction during growth can play a decisive role in the observed growth-front morphology, as has been proposed. ${ }^{11,12}$ A fast surface diffusion direction along the [ $1 \overline{1} 0$ ] direction in the $2 \times 4$ reconstructed surface will lead to a preferential island nucleation and to surfaces with a larger density of steps along that direction. Moreover, the higher reactivity of the step edges oriented along [110] enhances the 


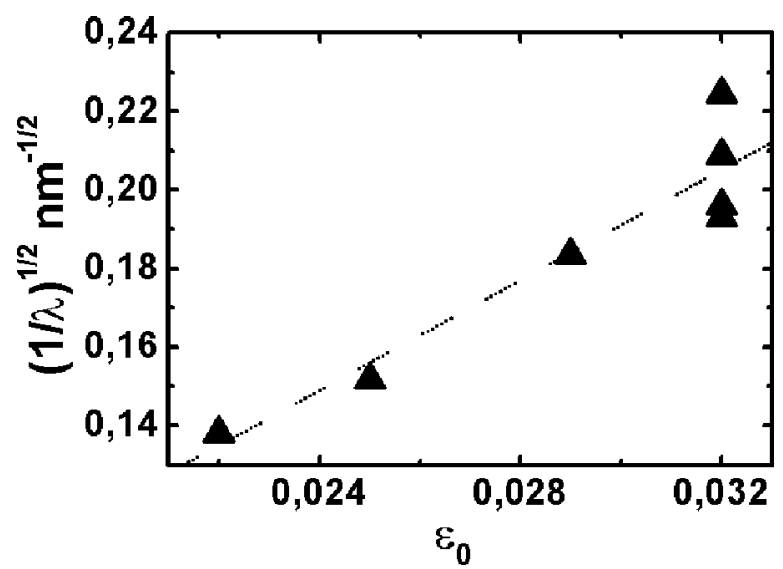

FIG. 3. Dependence of pitch period $\lambda$ on misfit strain $\epsilon_{0}$ for QWr structures formed in $\operatorname{In}_{x} \mathrm{Ga}_{1-x}$ As layers with different compositions ( $x=1,0.95,0.9$, and 0.85$)$ and thickness ( $d=2.5,5.8$, and $12 \mathrm{ML}$, respectively). Each point represents a different sample and the continuous line a linear fit according to the $\lambda \epsilon_{0}^{2}=K$ law.

an anisotropic morphology in the InP buffer layer could be responsible of the appearance of a periodic corrugation in the InAs layer as the best way for elastic strain relaxation. This idea is reinforced by the observed fact that QDs, instead of QWrs, are formed not only when InP buffer layers are grown by ALMBE [Fig. 1(a)], but also when InAs layers are deposited directly on the InP substrate, after desorbing the oxide, or on top of a very thin InP buffer layer $(8.5 \mathrm{~nm})$ grown by conventional MBE. In other words, nucleation of the QD would take place when the buffer-layer asymmetry has not been fully developed.

We would expect the QWr dimensions will depend on the misfit strain of the epilayer, given that strain relaxation is the driving mechanism for the formation of $3 \mathrm{D}$ structures. The misfit strain can be easily tuned by growing $\operatorname{In}_{x} \mathrm{Ga}_{1-x} \mathrm{As}$ layers of different compositions, as was described above. Our results show that QWr-like structures are also formed along [1 $1 \overline{10}$ ] after deposition of an appropriate amount of $\mathrm{In}_{x} \mathrm{Ga}_{1-x} \mathrm{As}$ and a subsequent anneal under $\mathrm{As}_{4}$. The dependence of the pitch period $\lambda$ on the lattice mismatch, $\epsilon_{0}$ can be observed in Fig. 3. These data can be fitted to an expression $\lambda \epsilon_{0}^{2}=K$, which is obtained from energy balance considerations of the surface roughness developed for stabilization of stressed surfaces. ${ }^{14,15}$

In conclusion, our results show that once there is an established anisotropy in the InP surface buffer layer, the 2D InAs layer transforms into periodic QWr-like structures to allow elastic strain relaxation. We have demonstrated that the period of the wires is determined by the misfit strain between the layer and the substrate. QWr-like structures capped with InP exhibit a strong PL emission at $1.55 \mu \mathrm{m}$ at RT. The emission is anisotropic with a degree of polarization around a $30 \%$.

The authors wish to acknowledge the Spanish 'CICYT', for financial support under Project No. TIC961020-C02.

${ }^{1}$ S. Fafard, Z. Wasilewski, J. McCaffrey, S. Raymond, and S. Charbonneau, Appl. Phys. Lett. 68, 991 (1996).

${ }^{2}$ A. Rudra, R. Houdré, J. F. Carlin, and M. Ilegems, J. Cryst. Growth 136, 278 (1994).

${ }^{3}$ H. Marchand, P. Desjardins, S. Guillon, J.-E. Paultre, Z. Bougrioua, R. Y.-F. Yip, and R. A. Masut, Appl. Phys. Lett. 71, 527 (1997).

${ }^{4}$ A. Ponchet, A. Le Corre, H. L'Haridon, B. Lambert, and S. Salaün, Appl. Phys. Lett. 67, 1850 (1995).

${ }_{5}^{5}$ J. Brault, M. Gendry, G. Grenet, and G. Hollinger, Appl. Phys. Lett. 73, 2932 (1998)

${ }^{6}$ L. González, Y. González, G. Aragón, M. J. Castro, M. L. Dotor, and D. J. Dunstan, J. Appl. Phys. 80, 3327 (1996).

${ }^{7}$ B. W. Wessels, J. Vac. Sci. Technol. B 15, 1056 (1997).

${ }^{8} \mathrm{~A}$ more detailed analysis of the optical properties measured in our structures is being developed and will be presented elsewhere.

${ }^{9}$ M. Taskinen, M. Sopanen, H. Lipsanen, J. Tulkki, T. Tuomi, and J. Ahopelto, Surf. Sci. 376, 60 (1997).

${ }^{10}$ M. A. Cotta, R. A. Hamm, T. W. Staley, S. N. G. Chu, L. R. Harriott, M. B. Panish, and H. Temkin, Phys. Rev. Lett. 70, 4106 (1993).

${ }^{11}$ M. D. Pashley, Phys. Rev. B 40, 10481 (1989).

${ }^{12}$ K. Shiraishi, Appl. Phys. Lett. 60, 1363 (1992).

${ }^{13}$ Y. Horikoshi, H. Yamaguchi, F. Briones, and M. Kawashima, J. Cryst. Growth 105, 326 (1990).

${ }^{14}$ D. J. Srolovitz, Acta Metall. 37, 621 (1989).

${ }^{15}$ A. J. Pidduck, D. J. Robbins, A. G. Cullis, W. Y. Leong, and A. M. Pitt, Thin Solid Films 222, 78 (1992). 